\title{
The MN1 oncoprotein activates transcription of the IGFBP5 promoter through a CACCC-rich consensus sequence
}

\author{
Magda A Meester-Smoor, Anco C Molijn, Yixian Zhao, Nicole A Groen, Cora A H Groffen, \\ Merel Boogaard, Diny van Dalsum-Verbiest, Gerard C Grosveld ${ }^{1}$ and Ellen C Zwarthoff
}

Department of Pathology, Erasmus MC-Josephine Nefkens Institute, PO Box 2040, 3000 CA Rotterdam, The Netherlands

${ }^{1}$ Department of Genetics and Tumor Cell Biology, St Jude Children's Research Hospital, 332 North Lauderdale, Memphis, Tennessee 38105, USA

(Requests for offprints should be addressed to E C Zwarthoff; Email: e.zwarthoff @erasmusmc.nl)

\begin{abstract}
The IGF-binding protein (IGFBP) family consists of six proteins that are expressed and secreted in different tissues. The proteins are regulators of physiological processes throughout the body by modulating the activity of IGF-I and IGF-II. In this article, we describe the coordinated expression of IGFBP5 and MN1 in meningiomas. MN1 is a transcriptional co-activator and we show that MN1 stimulates the IGFBP5 promoter in Hep3B cells. A CACCC-containing sequence, located 140 bp upstream of the transcription start site of the promoter, is required for MN1 action. This sequence matches with the CACCCAC consensus sequence that was selected in an oligonucleotide selection assay performed for MN1. The CACCC element has also been shown to be important for induction of the IGFBP5 promoter by retinoic acid (RA) and progesterone $(\mathrm{Pg})$. We were unable to confirm the effect of $\mathrm{Pg}$ on the promoter in Hep3B and U2-osteosarcoma cells regardless of the presence of $\mathrm{MN} 1$. On the other hand, we show that induction of the promoter by RA depends on co-expressed MN1 in Hep3B cells. MN1TEL, a leukemia-related fusion protein containing parts of the MN1 and TEL (ETV6) genes, is capable of stimulating the IGFBP5 promoter but is unable to cooperate with RA in Hep3B cells. This suggests that the effects of RA can be negatively affected in leukemias caused by MN1TEL.
\end{abstract}

Journal of Molecular Endocrinology (2007) 38, 113-125

\section{Introduction}

The insulin-like growth factor-binding proteins (IGFBPs) belong to a protein family with six members and are expressed and secreted in a tissue and developmental stage-specific manner. The proteins bind the small effector molecules IGF-I and IGF-II, and act as carrier proteins. IGFs are the most important regulators of mammalian growth and development and mediate the effects of growth hormone. The six IGFBP members are highly homologous, but each has distinct functions and biochemical properties. Dependent on the particular IGFBP and the type of tissue, the effect on IGF action can be inhibitory or stimulatory. IGFBPs can also have IGF-independent effects on cell growth. These effects are exerted by binding of IGFBPs to non-IGF molecules that are present either outside or inside the cell (Mohan \& Baylink 2002, Schneider et al. 2002, Bach et al. 2005). IGFBP5 is the most conserved member of the protein family and plays an important role in mediating IGF effects, particularly in bone. IGFBP5 binds IGF-I and is regulated by proteolysis, glycosylation, and phosphorylation. It binds extracellular matrix components in osteoblast cell cultures and potentiates IGF function by positioning it in the vicinity of the cell membrane (Andress \& Birnbaum 1992, Campbell \&
Andress 1997). In cultured osteoblasts and muscle cells, both inhibitory and stimulatory effects of IGFBP5 on IGF function have been described. Transgenic mice overexpressing IGFBP5 have osteopenia, lower body weight, retarded muscle development, and lower female fertility (Devlin et al. 2002, Salih et al. 2004). Within different tissues, there is a tight balance of IGFBP5 expression and disregulation of this equilibrium is thought to be a cause of disorders such as osteoporosis and renal osteodystrophy (Rosen \& Donahue 1998, Jehle et al. 2000). Other physiological processes in which IGFBP5 is involved are mammary gland involution, follicular development in the ovary, and kidney physiology (Schneider et al. 2002).

Expression of IGFBP5 is stimulated by IGF-1 through the phosphatidylinositol-3 kinase pathway (Kiepe et al. 2005). Transcription factors that have been shown to affect the IGFBP5 promoter are the progesterone receptor (PR; Boonyaratanakornkit et al. 1999), the retinoic acid receptor (RAR; Cesi et al. 2004, 2005), the glucocorticoid receptor (Gabbitas et al. 1996), activator protein-2 (AP-2) (Duan \& Clemmons 1995) and Myb (Tanno et al. 2002). Furthermore, prostaglandin E2, parathyroid hormone, and osteogenic protein-1 have been described to regulate the expression of IGFBP5 (Ji et al. 1999, Yeh \& Lee 2000, Erclik \& Mitchell 2005). 
Several studies have associated IGFBP5 expression with cancer. IGFBP5 was shown to be able to stimulate growth of prostate cancer cells in vitro and tumor growth in vivo (Miyake et al. 2000a,b). Both inhibition and induction of apoptosis have been described in breast cancer cells in vitro (Perks et al. 2000, Butt et al. 2003, 2005). Furthermore, IGFBP5 was shown to inhibit the proliferation of cervical carcinoma and osteosarcoma cells (Higo et al. 1997, Schneider et al. 2001). This study is, among others, based on our unpublished findings that IGFBP5 and the transcriptional co-activator MN1 are co-expressed in meningiomas, a benign brain tumor arising from the arachnoidal cap cells. This suggests that the expression of the two proteins is related.

The MN1 oncogene was cloned and described by our group in 1995 (Lekanne Deprez et al. 1995) on the basis of its involvement in a $t(4 ; 22)$ found in a meningioma. The protein consists of 1319 amino acids and has a nuclear localization. The primary protein sequence shows no homology to other proteins, but homologs of MN1 are found in mice (Mus musculus) and puffer fish (Tetraodon nigroviridus), proving conservation of MN1 in evolution (Meester-Smoor et al. 2005). Extensive searches in domain databases did not reveal any specific domain within the protein. The proline/glutamine-rich regions in the sequence suggested a function in transcription and indeed, we have shown that MN1 activates the transcription activity of the Moloney sarcoma virus long terminal repeat (MSV-LTR; Buijs et al. 2000). MN1 can synergize on this promoter with transcription mediated by the RAR-retinoic X receptor heterodimer (RXR) in the presence of the RAR-RXR ligand RA. We have also shown that MN1 binds p300 and RAC3, both known co-activators of RARs (van Wely et al. 2003). MN1 is fused to the TEL (ETVO) gene as a result of the $t(12 ; 22)$ in acute myeloid leukemia. The MN1TEL fusion protein is able to transform NIH3T3 cells in vitro and causes leukemia and lymphoid tumors in mice (Buijs et al. 2000, Kawagoe \& Grosveld 2005a,b).

Since $M n 1$ null mice die shortly after birth as the result of a cleft secondary palate, MN1 is an essential gene (Meester-Smoor et al. 2005). Interestingly, the development of several bones in the skull of these mice is abnormal. The affected bones are almost exclusively formed by intramembranous ossification. They are either completely agenic at birth or substantially thinner. Endochondral bone formation is normal. Thus, MN1 plays a crucial role in the formation of the membranous bones in the skull during mammalian development. This is further supported by Sutton $\mathrm{et} \mathrm{al}$. (2005). They have described the inhibition of osteoblastic cell proliferation by MN1 (Sutton et al. 2005).

In this report, we further investigated the role of MN1 in regulating transcription. Since MN1 has transcription-activating domains, we were prompted to investigate whether it can bind DNA and function as a transcription factor. We show that MN1 activates the IGFBP5 promoter, that this effect is exerted through CA-rich elements present in the proximal promoter, and that the activation of the promoter by RA is dependent on the presence of MN1 in the cells used in this study. These findings also explain the coordinated expression of MN1 and IGFBP5 in meningiomas.

\section{Materials and methods}

\section{Oligonucleotide-binding selection}

The method used has been described previously (van Wely et al. 2003) and is a modified version of that described by Blackwell \& Weintraub (1990). Oligonucleotides for selection contained a central 15-nucleotide random sequence flanked by two constant regions of $20 \mathrm{bp}$. Cellular extracts from MN1-expressing HtTA cells (Hela cells stably transfected with a tetracyclinecontrolled activator (HETA; van Wely et al. 2003)) were incubated with the oligonucleotides, and a monoclonal antibody against MN1 called 2F2 (Buijs et al. 2000, van Wely et al. 2003) was used to precipitate protein-DNA complexes. Bound DNA was amplified using the constant flanking sequences and used for further selection/enrichment. After five rounds of selection, PCR fragments were cloned and sequenced.

\section{Northern blot hybridization}

Total RNA was isolated from primary meningioma tumors using guanidinium thiocyanate method (Chirgwin et al. 1979). Electrophoresis, blotting, and hybridization were carried out using standard procedures as described previously (Lekanne Deprez et al. 1995). For detection of the MN1 transcript, a $0 \cdot 6 \mathrm{~kb}$ BamHI cDNA fragment was used. For IGFBP5, we used a $0.9 \mathrm{~kb}$ murine cDNA covering the entire coding region (Schuller et al. 1994). The coding regions of murine and human IGFBP5 are $91 \%$ identical. As a control for RNA loading, we used a $0.8 \mathrm{~kb}$ EcoRI-PstI fragment derived from the coding region of the human glyceraldehyde-3phosphate dehydrogenase (GAPDH) cDNA. Hybridization signals were quantified using a phosphorImager (GE Healthcare, Uppsala, Sweden). Hybridization signals for MN1 and IGFBP5 were corrected for background and GAPDH expression, and the resulting values were plotted against each other.

\section{Cloning of IGFBP5 promoter and generation of deletion/mutation constructs}

PCR was performed on bacterial artificial chromosomes (BAC) clone PR11-506C8 (Osoegawa et al. 1998) containing the IGFBP5 promoter. The forward primers 
are localized at position - 1111 (5'-CAGGTACCCTAGTGGCATGATTCGGTTC- ${ }^{\prime}$ ) and -204 of promoter (5'-CGGGTACC-GGAGGAGGGCGCTGTTCAGG-3 ${ }^{\prime}$ ) with respect to the transcription start site and each contains a KpnI site for cloning purposes. The reverse primer at position +1645 contains a BamHI site (5'-GCGGATCC-CAGGAGAGCGAGAGTGCAGG-3' ${ }^{\prime}$. The resulting PCR products were cloned in pGL2basic and sequenced. A KpnI-XmaI fragment $(-204$ to +119$)$ from pGL2 BP5 to $204+738$ was subcloned in pGL3basic, resulting in pGL3 BP5 $-204+119$. The mutants $\mathrm{mPR}$, dCA, dGT, and dCAdGT were generated using the QuikChange Site-Directed Mutagenesis Kit (Stratagene, La Jolla, CA, USA). The mPR mutant is as described by Boonyaratanakornkit et al. (1999). The CA-rich element at positions -147 to -133 (CCCCACCCCCACCCC) is mutated in CCCCACCaaaACCCC. The dCA mutant lacks the entire CA-rich element (from positions -147 to -133), and the dGT mutant lacks the complete GT-rich element localized at positions -87 to -78 (GGGTGTTGGG). The dCAdGT mutant lacks both elements. All plasmids were sequenced to confirm the different deletions/mutations.

\section{Cell culture and transfections}

Hela-derived HtTA cell lines and 3T3 Gene-Switch cell lines were generated and cultured as described previously (van Wely et al. 2003). In 3T3 Gene-Switch cell lines expression of HA-tagged-MN1 and HA-tag (as control) were induced overnight with mifepristone $\left(10^{-8} \mathrm{M}\right)$ and used to generate cell lysates for electrophoretic mobility shift assays (EMSAs). Transient transfections were performed using hepatoma cell line B (Hep3B; Knowles et al. 1980) or U2-osteosarcoma cells (U2OS). U2OS cells were obtained from the American Type Culture Collection (ATCG, Manassas, VA, USA) and are known as HTB-96 in this collection. Hep3B cells were maintained in $\alpha$-minimum essential medium $(\alpha$-MEM) supplemented with $5 \%$ fetal calf serum (FCS), $2 \mathrm{mM}$ L-glutamine, $1000 \mathrm{U} / 1$ penicillin, and $1 \mathrm{mg} / 1$ streptomycin at $37^{\circ} \mathrm{C}$ with $5 \% \mathrm{CO}_{2}$ and U2OS cells were cultured in Dulbecco's Modified Eagle's medium (DMEM) supplemented with $10 \%$ FCS, $1000 \mathrm{U} / 1$ penicillin, and $1 \mathrm{mg} / \mathrm{l}$ streptomycin at $37^{\circ} \mathrm{C}$ with $5 \% \mathrm{CO}_{2}$. The day before transient transfections cells were seeded in 24-well plates $\left(0 \cdot 7 \times 10^{5}\right.$ cells/well $)$. On the day of transfection, medium was changed and, if indicated in figure legends, progesterone $(\mathrm{Pg}(\mathrm{Q} 2600)$, Steraloids, Newport, RI, USA) or all-trans RA ((R2625), Sigma-Aldrich) was added at the desired concentration $\left(\mathrm{Pg}, 10^{-7} \mathrm{M}\right.$; RA, $\left.10^{-9} \mathrm{M}\right)$. Transfections were carried out using FuGENE 6 Transfection Reagent (Roche Applied Science) according to the manufacturer's recommendations. The MN1 expression plasmid, pMN50, has been described previously (van Wely et al.
2003), the expression plasmid for PR (pCMV3.1 PR- $\beta$ ) and the PR-responsive luc (PRE2-luc) reporter were obtained from Jenster et al. (1997) (Department of Urology, Erasmus MC, Rotterdam, The Netherlands). Expression plasmid pcDNA3 TEL was generated by cloning the insert of pSCTOP TEL (Buijs et al. 2000) (HindIII-NotI digestion) into pcDNA3.1. pcDNA3 MN1TEL was made by cloning the C-terminal region of MN1TEL (pSCTOP MN1TEL, SfiI-NotI digestion, (Buijs et al. 2000)) into pMN50. For a transfection experiment, $375 \mathrm{ng}$ expression plasmid and $25 \mathrm{ng}$ reporter plasmid were used. For each experiment, the total amount of transfected DNA and the molar ratio of CMV promoter were kept constant by the addition of puc6 plasmid DNA. After incubation for $48 \mathrm{~h}$, cells were lysed and luciferase activity was measured on a Fluoroscan Ascent FL luminometer (Thermo Electron Corporation, Waltham, MA, USA). Transfection efficiency was monitored regularly by adding a Renilla luciferase construct or a LacZ internal control plasmid (pcDNA3.1 HisLacZ). Transfection efficiency was shown not to vary within an experiment (data not shown). Each experiment was done at least thrice in triplicate.

\section{Real-time PCR (Taqman analysis)}

RNA was isolated from $3 \times 10^{5}$ cells using the Rneasy mini kit (Qiagen) according to the manufacturer's recommendations. RNA ( $3 \mu \mathrm{g})$ was reverse transcribed using 300 U Moloney Murine Leukemia Virus (M-MLV) reverse transcriptase (Invitrogen) and $750 \mathrm{ng}$ random hexamers. cDNA $(12.5 \mathrm{ng})$ was analyzed for the expression of RNA polymerase II, subunit A (PolR2A), MN1, and IGFBP5 by real-time PCR using SYBR green PCR assay (Applied Biosystems, Foster City, CA, USA) and an ABI Prism 7700. MN1 and IGFBP5 levels were corrected for the PolR2A levels detected in the samples. Levels of expression for MN1 and IGFBP5 were calculated relative to PolR2A level in the sample using the observed threshold cycle. Two separate RNA samples of cell lines were analyzed. Primers $(7.5 \mathrm{pmol}$ used for each PCR): PolR2A-F: 5'-CGGATGAACTGAAGCGAATG-3'; PolR2A-R: 5'-GAGTCCACAAGCAGTTTGG3'; MN1-F: 5'-TCGCTGATGGCAGAGCACAG-3; MN1-R: 5'-GTCATTCAAGTTAGGG-CAG-3'; IGFBP5-F: 5' -CGGGGGAGCCGAGAACAC-3'; and IGFBP5-R: 5'-GGCGCTGGCTGGAGTCGG-3'.

\section{Electrophoretic mobility shift assay (EMSA)}

Lysates were made of mifepristone $\left(10^{-8} \mathrm{M}\right)$-induced 3T3 cell lines (expressing hemaglutinin (HA) tagged MN1 and the HA-tag alone as a control) using EMSA lysis buffer (20 mM HEPES (pH 7.9), $10 \mathrm{mM} \mathrm{KCl,} 1 \mathrm{mM}$ $\mathrm{MgCl}_{2}, 300 \mathrm{mM} \mathrm{NaCl}, 0 \cdot 1 \%$ Triton X-100, 20\% glycerol, 
$0.5 \mathrm{mM}$ dithiothreitol (DTT); Roche). The cells were disrupted in a cuphorn sonifier. Protein concentration was measured and $20 \mu \mathrm{g}$ protein were used for each EMSA experiment. Protein lysates were incubated with 10000 c.p.m. ${ }^{32}$ P-labeled double-stranded oligo in $10 \mathrm{mM}$ HEPES (pH 7.9), $60 \mathrm{mM} \mathrm{KCl,} \mathrm{4 \%} \mathrm{Ficoll} \mathrm{400,}$ $1 \mathrm{mM}$ DTT, and $1 \mathrm{mM}$ EDTA at room temperature for $30 \mathrm{~min}$. Double-stranded oligos used for EMSA: IGFBP5CA: 5'-CCTCTCCCCACCCCCACCCCGTGTG-3'; IGFBP5-GT: $5^{\prime}$-GAGTTGGGTGTTGGGAAGCT-3' ${ }^{\prime}$; and Selex-78: 5'-GACCACCCACGTTGGCTCC-3'. Samples were analyzed on a 4\% acrylamide gel (375: 1; acrylamide: bisacrylamide) in $0.5 \times$ Tris-boric acidEDTA (TBE) at $15 \mathrm{~mA}$. After electrophoresis, the gel was transferred to paper and dried. Bands were quantified using a Typhoon 9200 scanner (GE Healthcare).

\section{Results}

\section{Oligonucleotide selection experiments reveal that MN1 recognizes a CACCC-rich sequence}

We have shown previously that MN1 can activate transcription from the MSV-LTR, and that MN1 contains a transcription-activating domain near its N-terminus (Buijs et al. 1995, van Wely et al. 2003). In order to investigate whether the MN1 protein can bind to DNA and whether it recognizes a specific DNA element, we performed an oligonucleotide selection assay. To this end, an oligonucleotide pool was synthesized with a random core of 15 residues with 20 residues of known flanking sequence on both sides. The pool was incubated with a lysate obtained from an HtTA HeLa cell line in which MN1 expression was induced by omitting tetracycline from the medium (van Wely et al. 2003). Oligonucleotides binding to MN1 were precipitated from the mixture using the MN1 monoclonal antibody 2F2. These oligonucleotides were amplified using primers annealing to the sequences flanking the random core. This selection procedure was repeated five times. The resulting oligonucleotides enriched for MN1-binding were cloned and the insert sequence of 54 different plasmids was determined. A CACCC-rich sequence was found in 34 plasmid inserts from which a consensus-binding sequence CACCCAC was deduced (Fig. 1). In seven plasmids, a sequence AGGTCAaAGGTCA resembling a RAR-RXR DR1binding site was observed. The RAR/RXR-responsive elements were shown to be responsible for a synergistic induction of expression by RA and MN1 in an earlier paper (van Wely et al. 2003). The remaining 13 inserts contained random sequences.

We have shown previously that MN1 and RAR-RXR can synergistically induce expression from the oligo ... GACNNNNNNNNNNNNNNNCTC....

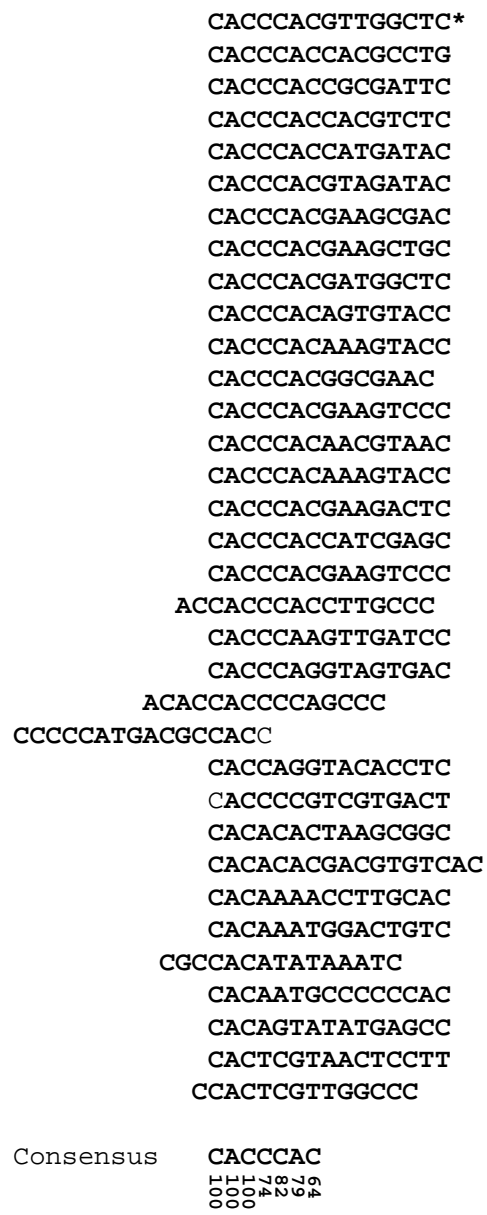

Figure 1 Oligonucleotide selection assay. To establish the consensus sequence CACCCAC, 34 oligonucleotides from the selection assay were used. The numbers indicate the percentage of oligonucleotides containing each nucleotide. The oligonucleotide indicated with an asterisk was used for the bandshift assay (Fig. 4).

MSV-LTR, a viral promoter that contains a RA-responsive element (van Wely et al. 2003). To investigate the effect of the CACCC element on expression, an oligonucleotide containing two of the CACCC consensus sequences was inserted into a reporter vector in front of a TATA box. However, when MN1 cDNA was co-transfected with the reporter, we were unable to establish an effect on the expression of this synthetic promoter.

\section{Coordinated expression of MN1 and IGFBP5 in meningiomas}

In a previous study, we showed that MN1 expression varies considerably between meningiomas (Lekanne Deprez et al. 1995). We also investigated a putative role 

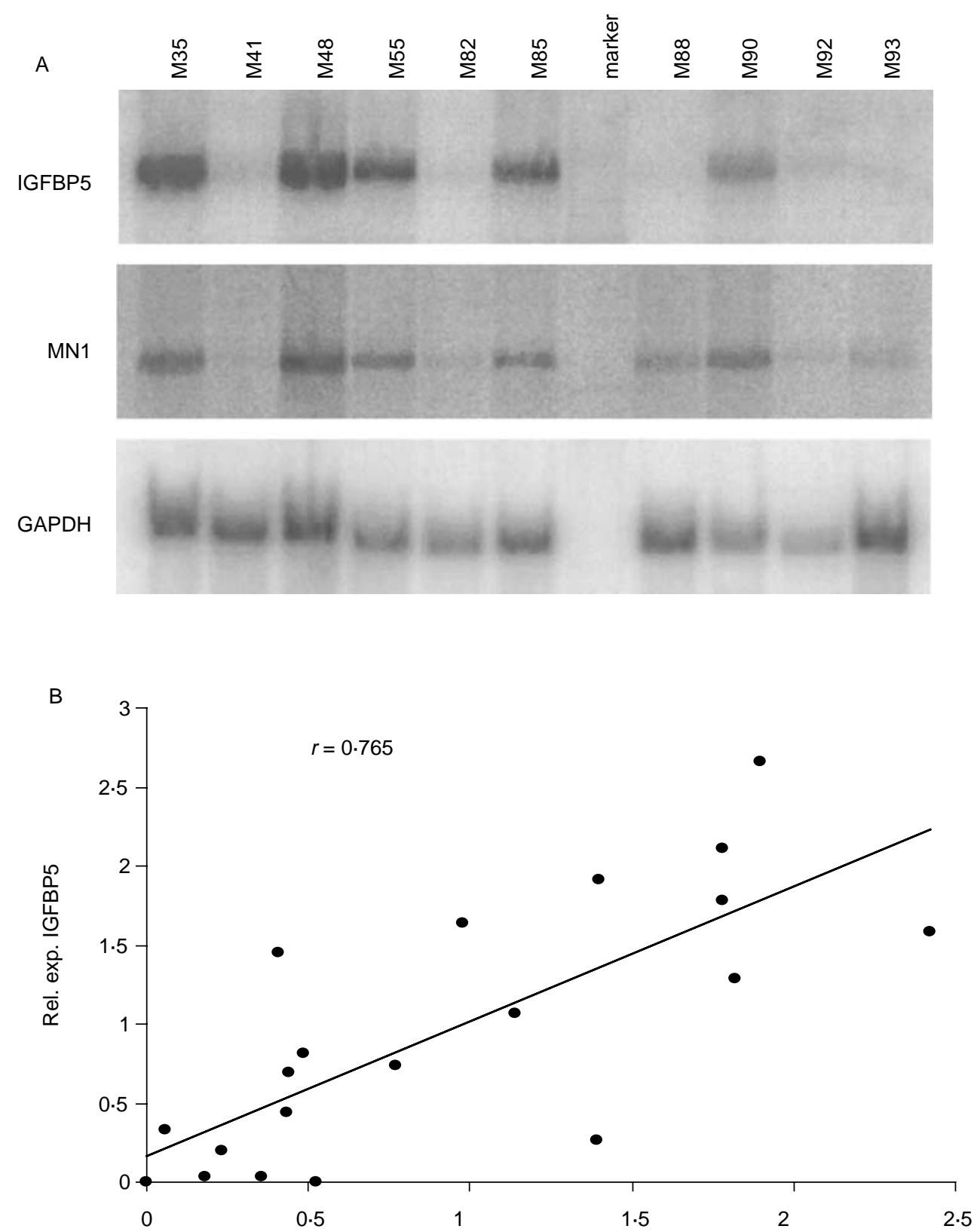

Rel. exp. MN1

Figure 2 Coordinated expression of MN1 and IGFBP5 in meningiomas. (A) The northern blot, containing $20 \mu \mathrm{g}$ of each RNA sample, was hybridized to probes for IGFBP5 (top panel), MN1 (middle panel), and GAPDH (lower panel). (B) Correlation of MN1 and IGFBP5 expression in 20 meningiomas. Expression of IGFBP5 and MN1 was calculated in relation to GAPDH using Phosphorlmager data. $r$, Pearson correlation.

for the IGF pathway in these benign tumors. These experiments showed that there was a correlation between the expression of MN1 and IGFBP5. Figure 2A shows a representative northern blot containing RNA from ten meningiomas hybridized with probes against IGFBP5 (top panel), MN1 (middle panel), and control
GAPDH (bottom panel). Quantification of expression of MN1, IGFBP5, and GAPDH using a phosphorImager revealed that the expression of the $M N 1$ gene correlated with the expression of the IGFBP5 gene in 18 out of the 20 meningiomas tested, with high expression of MN1 occurring in the same tumors that 
showed high expression of IGFBP5. Figure 2B is a graphical representation of all the meningiomas tested; the Pearson correlation coefficient $(r)$ calculated is $0 \cdot 765$ ( $P$ value $<0 \cdot 0001$ at significance level $0 \cdot 01)$. These results suggest a functional relationship between the expression of these genes.

\section{MN1 stimulates expression of the IGFBP5 gene through CA- and GT-rich elements}

Inspection of the promoter of the IGFBP5 gene showed that it contains a CA-rich element that may present a possible natural target for regulation of expression by MN1. Another element is GT-rich, and is nearly the

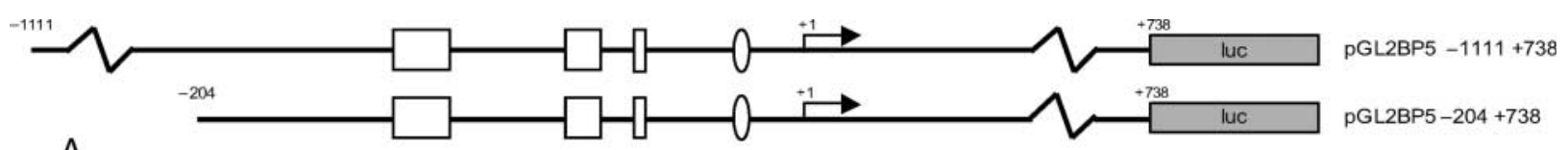

A
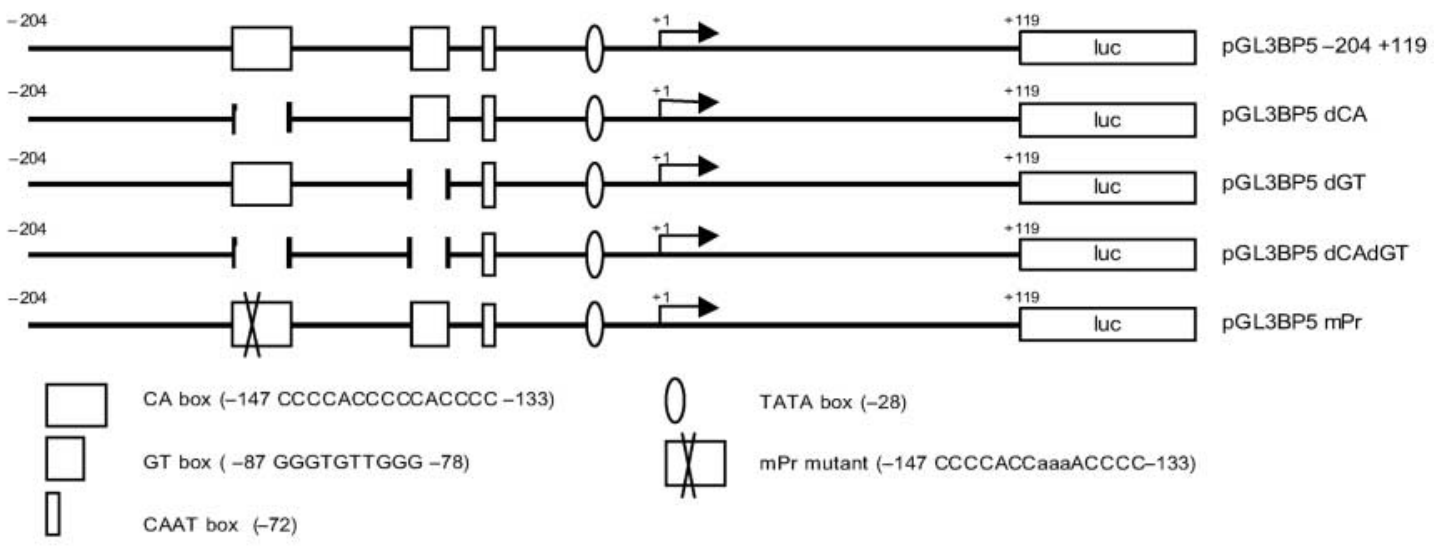

B

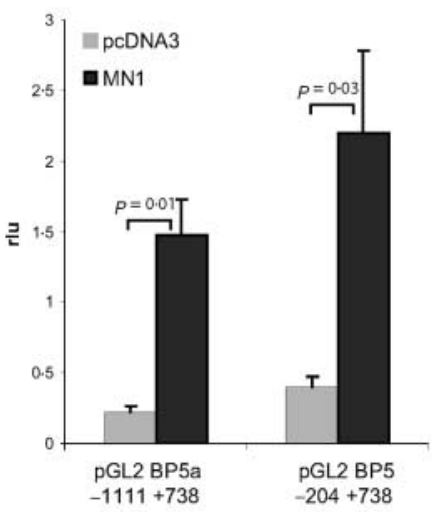

C

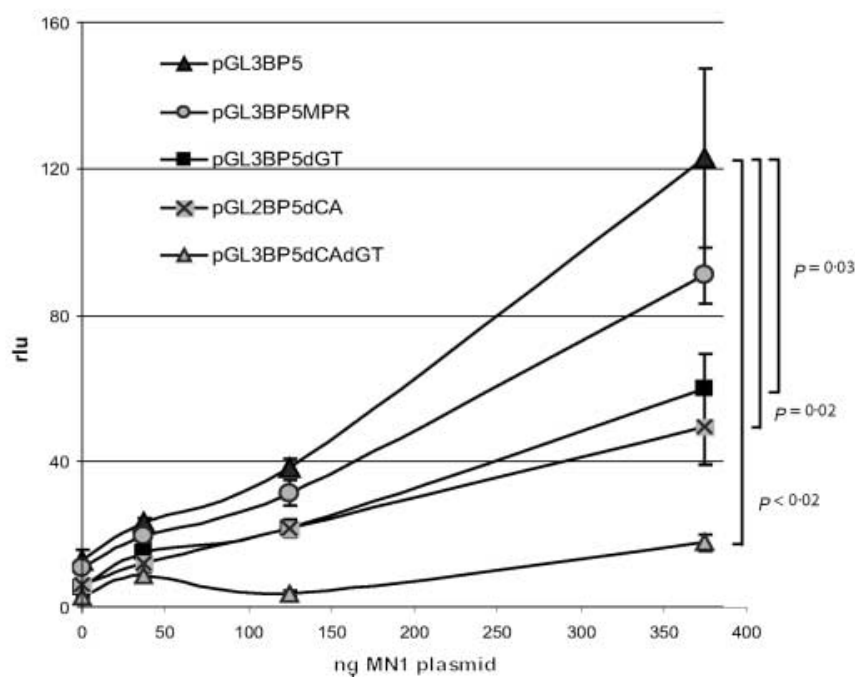

Figure 3 MN1 stimulates the IGFBP5 promoter through CA-rich elements. (A) Schematic representation of luciferase reporter constructs of the IGFBP5 promoter. Numbers indicate nucleotide positions relative to the transcription start site $(+1$, indicated with an arrow). The constructs of the IGFBP5 promoter contain +738 or +119 basepairs of untranslated exon 1 . The constructs used for Fig. 3B are cloned in pGL2basic. The constructs used for Fig. 3C are cloned in pGL3basic. This vector contains a modified coding region in the luciferase gene, resulting in an enhanced luciferase activity. (B) In Hep3B cells, 25 ng reporter plasmids and 120 ng MN1 expression plasmid or pcDNA3 were transfected. MN1 stimulates the IGFBP5 promoter and -204 bp of the promoter is sufficient for induction by MN1. (C) In Hep3B cells, 25 ng reporter plasmid and increasing amounts of MN1 expression plasmid were transfected. The promoter is stimulated in a dose-dependent manner and the mutated promoters are stimulated less efficiently. Values shown are the mean of triplicate wells \pm s.E., except for Fig. $3 C$ (mean of two separate RNA samples analyzed \pm s.E.). $P$ values are shown for significant changes (two-tailed Student's $t$-test). rlu, relative light units. 
reverse sequence of the consensus sequence picked up by the oligonucleotide selection assay. Both the elements are located just upstream of the transcription start site: (-146) CCCCACCCCACCC $(-132)$ and $(-86)$ GGTGTTGGG $(-77)$.

In order to establish whether MN1 induces expression of IGFBP5, we cloned the promoter in front of a luciferase reporter (outline of the constructs is shown in Fig. 3A). Transient transfections were carried out using an MN1 expression construct. For these experiments, we used the Hep3B liver carcinoma cell line, in which there is no expression of the endogenous $M N 1$ gene. Figure 3B shows that MN1 induces the IGFBP5 promoter about sixfold, and that the smaller promoter construct spanning nucleotide (nt) -204 to nt 738 is sufficient for induction by MN1. Deletion of nucleotide +119 to +738 did not influence the induction of MN1 (data not shown).

To investigate the role of the CA and/or GT-rich elements in MN1-induced expression, the sequences were deleted from the shortest IGFBP5 promoter construct. Both single and double mutants (lacking both CA and GT-rich elements) were generated. The effect of the mutations is shown in Fig. 3C. MN1 was able to induce the IGFBP5 promoter in a dosedependent manner, and deletion of both elements negatively affected the induction of the IGFBP5 promoter by MN1. When both elements were deleted, promoter activity was almost completely abolished. In the literature, the CA-rich element is described as a PR-sensitive element (Boonyaratanakornkit et al. 1999). A mutant used by the authors of this article in which 3 Cs were exchanged with As was no longer able to confer PR activity to the IGFBP5 promoter. We used this mutant to investigate a possible interplay between $\mathrm{MN1}$ and PR. Figure 3C shows that the mPR mutation construct is also stimulated less efficiently by MN1. On the basis of these experiments, we conclude that MN1 is able to efficiently induce the IGFBP5 promoter and that the CA- and GT-rich elements are involved in MN1induced expression.

\section{MN1 binds indirectly to CA-containing oligonucleotides}

The finding that MN1 recognizes a CACCC sequence in the oligonucleotide selection experiments and is able to activate the IGFBP5 promoter containing similar elements prompted us to investigate whether MN1 binds to the CA- and GT-rich DNA elements in a bandshift assay. To this end, an NIH3T3 cell line was used in which the expression of HA-tagged MN1 can be induced by addition of mifepristone. Lysates of $\mathrm{HA}$ and HA-MN1 cell lines were incubated with ${ }^{32} \mathrm{P}$-labeled double-stranded oligonucleotides containing the CA consensus sequence (as shown in Fig. 4A; selex 78) and
CA- and GT-containing oligonucleotides derived from the IGFBP5 promoter sequence (see Materials and methods section and Fig. 4B for specific sequences). The results of the mobility shift assays are shown in Fig. 4. The CA-containing selex oligonucleotide (selex 78) was found to bind to four separate protein complexes (lane 4, complexes numbered I-IV). Figure $4 \mathrm{~B}$ is a graphical representation of the binding. The four complexes are distinguishable as four peaks. The CA oligonucleotide derived from the IGFBP5 promoter (IGFBP5-CA) binds two complexes, similar to complexes I and II detected with selex 78 oligonucleotide (lane 1). Although the sequence of the GT oligonucleotide is not exactly complementary to the CCACCC consensus sequence, the IGFBP5-GT oligonucleotide binds two complexes similar to complexes III and IV. Close examination of the sequences of the selex 78 and the IGFBP5-GT oligonucleotide reveals that the oligonucleotides share the GTTGG sequence: most likely, this represents the sequence responsible for the complexes binding to both oligonucleotides.

Binding specificity was established by competition with cold oligonucleotides (lane 3, 6, and 9). In the presence of HA-MN1, the relative intensities of complexes I and II change and complex II is now the major complex for bandshifts with both selex 78 and IGFBP5-CA oligonucleotides experiments (lane 2 and 5; indicated with arrows in Fig. 4A and B). Possible explanations are that the newly formed complex with HA-MN1 migrates at the same position as the complex II that is seen in the absence of MN1, or alternatively, that MN1 stimulates the formation of complex II, for instance by increasing the recruitment of proteins, such as p160 and p300 that bind to MN1. No influence of HA-MN1 was detectable on the complexes binding the IGFBP5-GT oligonucleotide.

In order to establish whether binding of MN1 was direct or indirect, mobility shift assays were performed with MN1 produced through in vitro transcriptiontranslation. No binding of MN1 to the oligonucleotides was found (data not shown). Thus, we conclude that MN1 recognizes CACCC-rich sequences indirectly, presumably by binding to a CACCC-specific transcription factor.

\section{The progesterone receptor (PR) does not stimulate the IGFBP5 promoter in Hep3B cells and U2 osteo- sarcoma cells (U2OS) cells, and the effect of MN1 is not affected by PR}

It has previously been shown by Boonyaratanakornkit et al. (1999) that the PR can stimulate the IGFBP5 promoter in the presence of its ligand, progesterone. Although no canonical PR-responsive elements are present in the IGFBP5 promoter, Boonyaratanakornkit et al. (1999) showed that induction by PR is mediated by 


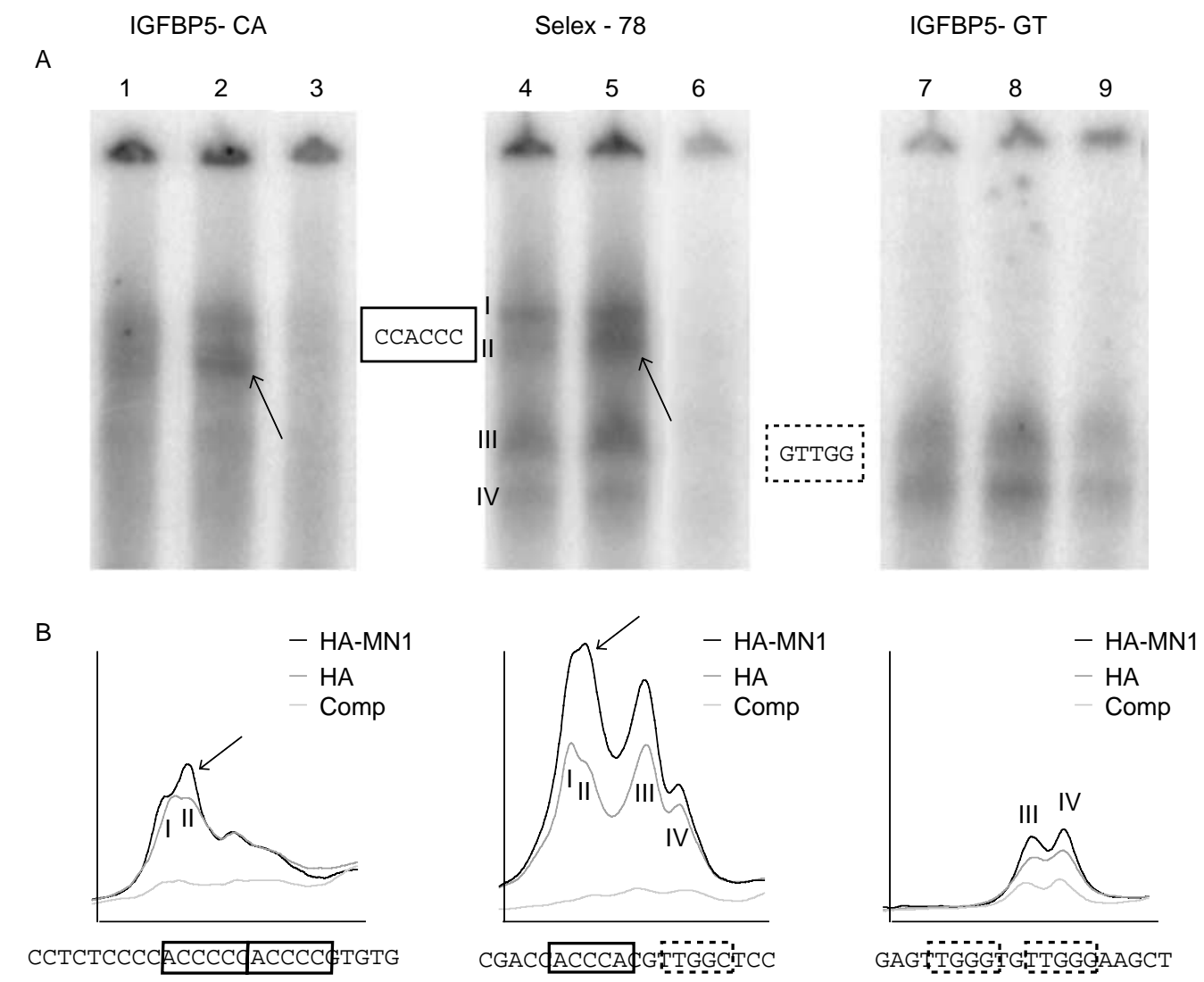

Figure 4 Bandshift assay using the selected CACCCAC sequence and IGFBP5-derived oligonucleotides. (A) Autoradiograms of bandshift assays using three different oligonucleotides incubated with total lysates of cell lines expressing HA-tagged MN1 and the HA-tag alone as a control. Four protein complexes binding the selex78 oligonucleotide are indicated with roman numbers I-IV. Lanes 1, 4, and 7: shifts observed with control lysate of HA-expressing 3T3 cell line. Lanes 2, 5, and 8: shifts observed with lysate of HAMN1-expressing cell line. Arrows indicated the more intense band appearing in lanes 2 and 5. Lanes 3, 6, and 9: competition experiment using cold oligonucleotides (100× molar excess). (B) Graphical representation of bandshift assays. Peaks are indicated for protein complexes I, II, III, and IV. More intense bands are indicated with an arrow.

the CACCC element in the promoter. Since MN1 also uses this element to induce to IGFBP5 promoter, and can collaborate with another nuclear receptor, the RARRXR heterodimer (van Wely et al. 2003), we sought to investigate the effect of a combination of PR and MN1 on this promoter. To this end, we co-transfected MN1 and PR expression constructs and the IGFBP5 reporter into Hep3B cells. The result of this experiment is shown in Fig. 5A. Whereas MN1 efficiently induced the IGFBP5 promoter as shown above, we were unable to find induction of the promoter by PR. Neither did different concentrations of PR result in induction of IGFBP5 in Hep3B cells (data not shown). Co-transfection of MN1 and PR did not increase the induction of the IGFBP5 promoter by MN1.

Since the experiments described by Boonyaratanakornkit et al. (1999) had been carried out in human U2OS cells, we repeated the experiments in this cell line. U2OS cells express MN1 endogenously as is shown in Fig. 5C and, as a consequence, no effect of transfection of $\mathrm{MN1}$ is obvious (Fig. 5B). Figure 5C also shows that MN1 and IGFBP5 are both expressed in U2OS cells but not in Hep3B cells. In contrast to Boonyaratanakornkit et al. (1999), we found inhibition of IGFBP5 by the PR in U2OS cells rather than stimulation (Fig. 5B). As a control for PR activity, we established that PR in the presence of ligand but not in its absence could induce a luciferase reporter driven by two copies of the canonical progesterone-responsive element (PRE, Fig. 5D). The results are shown for U2OS cells and were similar in Hep3B cells.

\section{The IGFBP5 promoter can also be induced by MN1TEL, and induction by MN1 can be enhanced by RA in Hep3B cells}

Because of the effect of IGFBP5 in various forms of cancer, we next sought to investigate whether the leukemia-associated MN1TEL fusion protein is also capable of induction of the IGFBP5 promoter. Figure 5E 

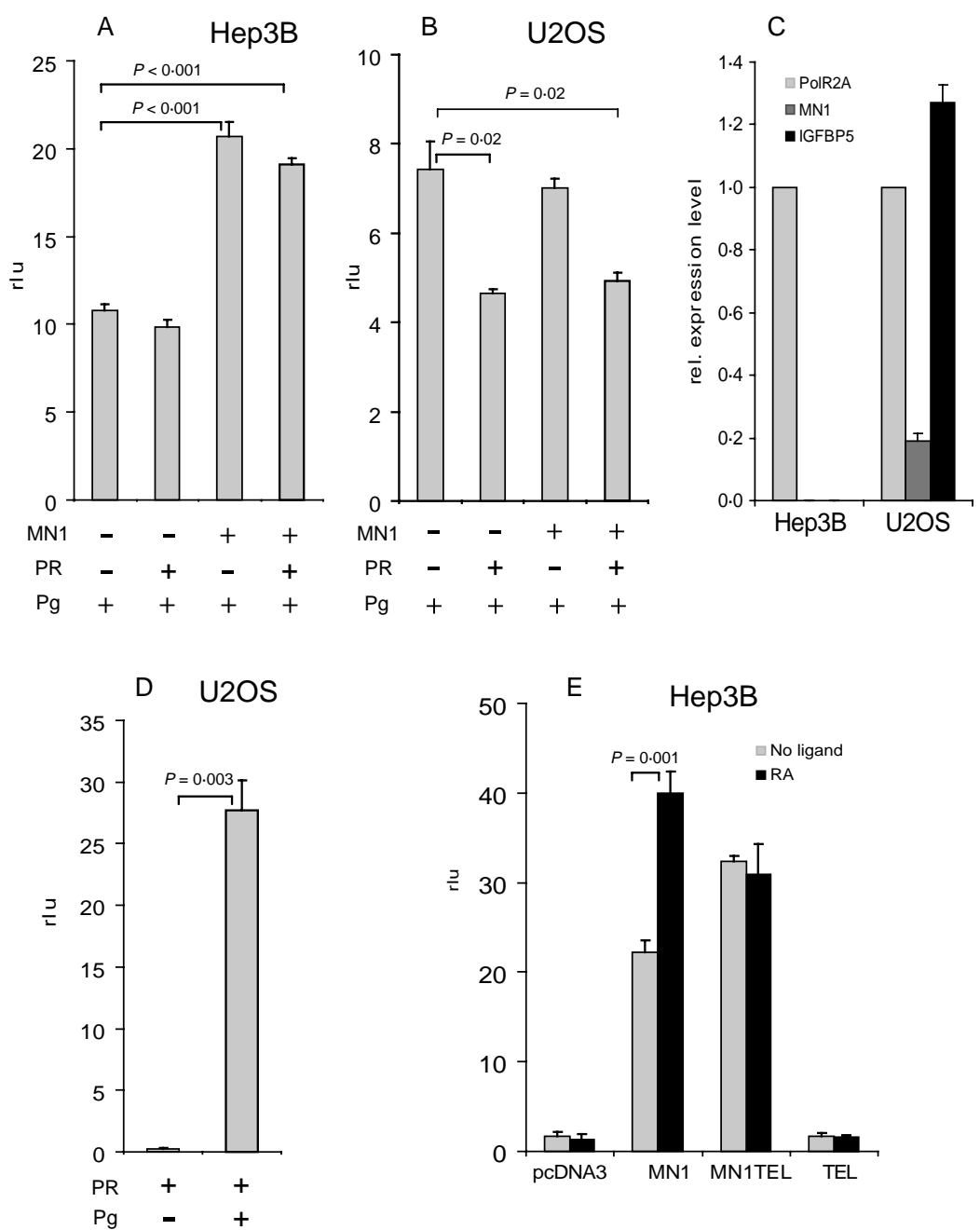

Figure 5 The PR does not stimulate the IGFBP5 promoter; RA induction of IGFBP5 depends on the presence of MN1. (A) Expression plasmids for MN1 (125 ng) and PR (250 ng) were transfected together with pGL3 BP5 - 204+119 in Hep3B cells in the presence of progesterone $\left(\mathrm{Pg} ; 10^{-7} \mathrm{M}\right)$. The PR has no effect on the transfected IFGBP5 promoter. (B) Transfection performed in U2OS cells (as described for $5 \mathrm{~A}$ ). The PR does not stimulate transcription from the transfected IFGBP5 promoter in U2OS cells. (C) Endogenous expression of MN1 and IGFBP5 in Hep3B and U2OS cells as determined by real-time PCR. Expression of MN1 and IGFBP5 was observed in U2OS cells, whereas Hep3B had no expression of either of the genes. (D) Control for the effect of progesterone. pPRE-luc contains two copies of the canonical PR-responsive element and, when co-transfected with $P R$, is efficiently induced in the presence of $\mathrm{Pg}$. (E) In Hep3B cells, $25 \mathrm{ng}$ reporter plasmid (pGL3 BP5 -204+119) and $375 \mathrm{ng}$ expression plasmid for MN1, MN1TEL, and TEL were transfected in the presence or absence of retinoic acid (RA; $\left.10^{-9} \mathrm{M}\right)$. RA can stimulate the IGFBP5 promoter only in the presence of MN1. MN1TEL cannot replace MN1 in this process. TEL does not have any effect on the IGFBP5 promoter. Values shown are the mean of triplicate wells \pm S.E. $P$ values are shown for significant changes (two-tailed Student's $t$-test). rlu, relative light units; RA, retinoic acid; PR, progesterone receptor; Pg, progesterone.

shows that MN1TEL is indeed able to induce the IGFBP5 promoter. It has also been described that RA can induce IGFBP5 in neuroblastoma cells and other cell types (Cesi et al. 2004, 2005). Since MN1 is known to collaborate with the receptors of this ligand, RAR-RXR, by inducing expression from the MSV-LTR (van Wely et al. 2003), we investigated the effect of RA on the IGFBP5 promoter in the presence or absence of MN1. In Hep3B cells that express all RARs and RXRs (Wan et al. 1998), we observed that addition of RA alone is 
insufficient to stimulate the promoter. In the presence of MN1, however, a clear induction was found (Fig. 5E). Thus, in Hep3B cells, induction of the IGFBP5 promoter with RA requires expression of MN1.

Since we previously found that the fusion protein MN1TEL can induce expression, but is not capable of synergising with RA in the case of the MSV-LTR (van Wely et al., unpublished observations), we investigated whether the same was true for the IGFBP5 promoter. In analogy to our results with the MSV-LTR, the effect of MN1TEL was not enhanced in the presence of RA. We also found that the fusion partner TEL had no effect on the IGFBP5 promoter (Fig. 5E). If induction of the IGFBP5 promoter by RA was always dependent on co-expressed MN1, the sole addition of RA to U2OS cells would be sufficient to induce the IGFBP5 promoter, since these cells express MN1 endogenously. Transfection of the IGFBP5 reporter construct in U2OS cells and incubation with RA, however, did not result in increased expression from the promoter. We conclude that there was no induction of the IGFBP 5 promoter by RA alone either in Hep3B or in U2OS cells and that expression of MN1 in Hep3B cells is required for RA to have an effect on the IGFBP5 promoter.

\section{Discussion}

The oligonucleotide selection experiments revealed a consensus sequence CACCCAC that was recognized by protein complexes containing MN1. Our data suggest that this binding is presumably indirect and through another, as yet unknown, protein or proteins. The CACCCAC sequence is present in the list of regulatory motifs in human promoters generated by the study of Xie et al. (2005), in which over 17000 well-annotated genes were studied by aligning promoters and $3^{\prime}$ UTRs of different mammalian species. The motif CACCCAC was found in reverse (GTGGGTGK) together with about 150 other short regulatory sequences. Searching the Transfac database with the motif in both orientations shows that many transcription factors, such as AP2-1, Egr-1, paired box protein (PAX) 1, 5 and 9, Sp1, AP1, human Tcell leukemia virus enhancer factor (HLTF), and Dopamine receptor regulating factor (DRRF) bind to this motif (http://www.gene-regulation.com/pub/ databases.html\#transfac). In addition, there is an intriguing correlation between the CACCCAC-MN1binding motif and the GACCACCCAC elements to which GLIoma-associated oncogene (GLI) 1, 2, and 3 and the family members GLIS 1, 2, and 3 bind (Kim et al. 2003, Kasper et al. 2006). In the sequences that were determined on the basis of the oligonucleotide selection assay, the same flanking sequence was present upstream of the conserved motif in all cases. If the motif is then extended at its $5^{\prime}$ end with three nucleotides of flanking sequence, it reads GACCACCCAC. The sequence of the other flanking sequence is different for all selected sequences, suggesting that a selection has occurred for one of the two flanking sequences in conjunction with the core motif CACCCAC, and thus that it is also possible that a GLI family member is a target protein for MN1.

MN1 and IGFBP5 are co-expressed in meningiomas, a benign tumor of the meninges. Considering the results presented in this work, the most likely explanation for this finding would be that MN1 induces IGFBP5 expression in these tumors. Expression of MN1 and IGFBP5 between the different meningiomas differs considerably, some tumors having hardly detectable expression levels, and others having high expression. Our comparison of expression levels of MN1 and IGFBP5 with other characteristics of these meningiomas produced no correlation with the position of the tumor, histological subtype or grade, nor any evidence for involvement of the NF2 tumor suppressor gene (Lekanne Deprez et al. 1995, Kros et al. 2001). Nordqvist \& Mathiesen (2002) studied the expression of IGFBP5 in three groups of meningiomas (classified on the basis of invasiveness) and concluded that higher expression of IGFBP5 is observed predominantly in tumors that do not invade the brain. This would correlate with our finding, since, in meningiomas, invasion of surrounding structures is extremely rare, and was certainly not seen in the meningiomas in our study. Thus, both studies suggest that the expression of IGFBP5 is high in some meningiomas and low in others. Whether expression is higher than normal is not known at present. Persistent, but low-level expression of IGFBP5 mRNA and protein has been observed in the mouse meninges (van Kleffens et al. 1999), but there is no data on the IGFBP5 expression level in normal human meninges.

Boonyaratanakornkit et al. (1999) reported previously that PR efficiently stimulated the IGFBP5 promoter, and that the CA-rich region was responsible for this, although the sequence does not resemble a canonical PRE. They also showed that the PR does not bind the CA element directly. We investigated the relationship between MN1 and $\mathrm{PR}$ in the presence of $\mathrm{Pg}$ and we were not able to reproduce the effect of PR on the IGFBP5 promoter either in Hep3B or in U2OS cells. In both cell lines, the control PRE-driven reporter was efficiently induced by co-transfected PR after the addition of Pg. Explanations for these discrepancies are difficult to give, but may result from differences in cell lines after prolonged culturing or in culture conditions.

In the neuroblastoma cell line LAN-5, cervical carcinoma cells, human breast carcinoma cells, and rat osteoblastic cells, RA was shown to stimulate the expression of IGFBP5 (Dong \& Canalis 1995, Higo et al. 1997, Cesi et al. 2004, 2005). Mutagenesis of the CACCC element decreased, but did not completely abolish RA induction in LAN-5. This suggests that other sites for RA 


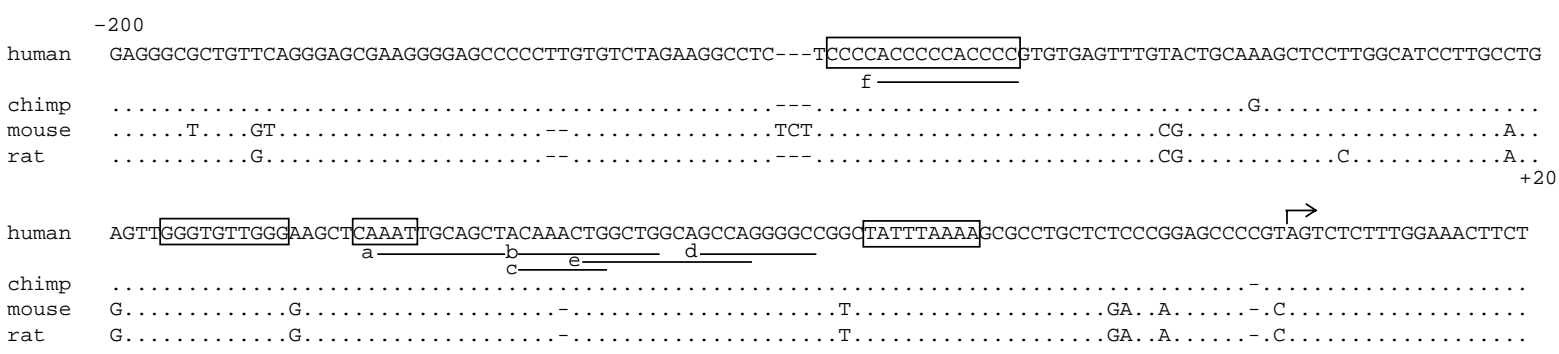

Figure 6 Alignment of the IGFBP5 promoter in human, chimp, mouse, and rat. The promoter is aligned for bp -200 to +20 relative to the transcription start site (indicated with an arrow). The CA- and GT-rich elements, CAAT box, and TATA box are boxed. Known binding sites are indicated under the sequence with a line. $a=E-$ box, CCAAT/enhances binding protein (C/EBP)-responsive element (RE); b, cortisol and osteogenic protein $1 \mathrm{RE}$; c, Myb-binding site; d, AP2-binding site; e, nuclear factor-1 (NF1)-binding site; f, MN1 $\mathrm{RE} /$ progesterone RE.

induction are present in the promoter (Cesi et al. 2004, 2005). In contrast, RA decreased IGFBP5 levels in the prostate adenocarcinoma cell line PC-3 and in the human breast carcinoma cell line T47D (Shemer et al. 1993, Hwa et al. 1997). In the Hep3B and U2OS cells studied in this work, RA had no effect on its own. We showed that RA can cooperate with exogenously added MN1 in Hep3B cells, leading to stimulation of the promoter, but cannot do this in U2OS cells that express the protein endogenously. These results suggest that regulation of the IGFBP5 promoter by RA occurs, but that unknown cell-dependent factors determine the outcome.

Interestingly, MN1TEL was as efficient in activating the IGFBP5 promoter as MN1, but this activity could not be enhanced by addition of RA. TEL on its own had no effect on the promoter. Since MN1TEL is under control of the MN1 promoter, its expression is probably similar to expression of $\mathrm{MN1}$, which makes it possible that the fusion protein competes for MN1-binding partners. Its inertness in collaborating with RA is not unique for the IGFBP5 promoter. The Moloney sarcoma virus long terminal repeat (MSV-LTR) is stimulated by both MN1 and MN1TEL; on this promoter too, MN1 can collaborate with RA, whereas MN1TEL cannot. This property of MN1TEL could play a role in leukemia caused by MN1TEL.

No other transcription factors have been described that regulate the transcription of the IGFBP5 gene through the regions identified as being important for MN1. The CACCCAC motif overlaps with two putative AP-2 sites but they were shown not to be used by this transcription factor. Instead, a more proximal GCCNNNGGC sequence within the promoter was shown to be the target of AP-2. Both the CA- and the GT-rich motifs are highly conserved between species. Figure 6 shows the alignment of the IGFBP5 promoter, which ranges from nt -200 to +20 for human, chimp, mouse, and rat. Proven consensus sequences for several factors are indicated. Most of them cluster in the region between the CAAT box and the TATA box. The sequences identified here to be important for proper
MN1 function are completely conserved, indicative of an important role for these regions of the promoter. Future experiments should reveal whether transcription factors known to bind CA-rich regions, such as Sp1, GLI etc., regulate the transcription of the IGFBP5 promoter and how MN1 is involved in this regulation.

\section{Acknowledgements}

This work was supported by the Dutch Cancer Society (grant 1998-1778 and 2003-2869). The authors declare that there is no conflict of interest that would prejudice the impartiality of this scientific work.

\section{References}

Andress DL \& Birnbaum RS 1992 Human osteoblast-derived insulinlike growth factor (IGF) binding protein-5 stimulates osteoblast mitogenesis and potentiates IGF action. Journal of Biological Chemistry 267 22467-22472.

Bach LA, Headey SJ \& Norton RS 2005 IGF-binding proteins-the pieces are falling into place. Trends in Endocrinology and Metabolism 16 228-234.

Blackwell TK \& Weintraub H 1990 Differences and similarities in DNAbinding preferences of MyoD and E2A protein complexes revealed by binding site selection. Science 250 1104-1110.

Boonyaratanakornkit V, Strong DD, Mohan S, Baylink DJ, Beck CA \& Linkhart TA 1999 Progesterone stimulation of human insulin-like growth factor-binding protein-5 gene transcription in human osteoblasts is mediated by a CACCC sequence in the proximal promoter. Journal of Biological Chemistry 274 26431-26438.

Buijs A, Sherr S, van Baal S, van Bezouw S, van der Plas D, Geurts van Kessel A, Riegman P, Lekanne Deprez R, Zwarthoff E, Hagemeijer A et al. 1995 Translocation $(12 ; 22)$ (p13;q11) in myeloproliferative disorders results in fusion of the ETS-like TEL gene on 12p13 to the MN1 gene on 22q11 (published erratum appears in Oncogene 1995 Aug 17;11(4):809). Oncogene 10 1511-1519.

Buijs A, van Rompaey L, Molijn AC, Davis JN, Vertegaal AC, Potter MD, Adams C, van Baal S, Zwarthoff EC, Roussel MF et al. 2000 The MN1TEL fusion protein, encoded by the translocation $(12 ; 22)$ (p13;q11) in myeloid leukemia, is a transcription factor with transforming activity. Molecular and Cellular Biology 20 9281-9293. 
Butt AJ, Dickson KA, McDougall F \& Baxter RC 2003 Insulin-like growth factor-binding protein-5 inhibits the growth of human breast cancer cells in vitro and in vivo. Journal of Biological Chemistry 278 29676-29685.

Butt AJ, Dickson KA, Jambazov S \& Baxter RC 2005 Enhancement of tumor necrosis factor-alpha-induced growth inhibition by insulinlike growth factor-binding protein-5 (IGFBP-5), but not IGFBP-3 in human breast cancer cells. Endocrinology 146 3113-3122.

Campbell PG \& Andress DL 1997 Insulin-like growth factor (IGF)binding protein-5-(201-218) region regulates hydroxyapatite and IGF-I binding. American Journal of Physiology 273 E1005-E1013.

Cesi V, Vitali R, Tanno B, Giuffrida ML, Sesti F, Mancini C \& Raschella G 2004 Insulin-like growth factor binding protein 5: contribution to growth and differentiation of neuroblastoma cells. Annals of the New York Academy of Sciences 1028 59-68.

Cesi V, Giuffrida ML, Vitali R, Tanno B, Mancini C, Calabretta B \& Raschella G 2005 C/EBP alpha and beta mimic retinoic acid activation of IGFBP-5 in neuroblastoma cells by a mechanism independent from binding to their site. Experimental Cell Research 305 179-189.

Chirgwin JM, Przybyla AE, MacDonald RJ \& Rutter WJ 1979 Isolation of biologically active ribonucleic acid from sources enriched in ribonuclease. Biochemistry 18 5294-5299.

Devlin RD, Du Z, Buccilli V, Jorgetti V \& Canalis E 2002 Transgenic mice overexpressing insulin-like growth factor binding protein-5 display transiently decreased osteoblastic function and osteopenia. Endocrinology 143 3955-3962.

Dong Y \& Canalis E 1995 Insulin-like growth factor (IGF) I and retinoic acid induce the synthesis of IGF-binding protein 5 in rat osteoblastic cells. Endocrinology 136 2000-2006.

Duan C \& Clemmons DR 1995 Transcription factor AP-2 regulates human insulin-like growth factor binding protein-5 gene expression. Journal of Biological Chemistry 270 24844-24851.

Erclik MS \& Mitchell J 2005 Activation of the insulin-like growth factor binding protein- 5 promoter by parathyroid hormone in osteosarcoma cells requires activation of an activated protein-2 element. Journal of Molecular Endocrinology 34 713-722.

Gabbitas B, Pash JM, Delany AM \& Canalis E 1996 Cortisol inhibits the synthesis of insulin-like growth factor-binding protein-5 in bone cell cultures by transcriptional mechanisms. Journal of Biological Chemistry 271 9033-9038.

Higo H, Duan C, Clemmons DR \& Herman B 1997 Retinoic acid inhibits cell growth in HPV negative cervical carcinoma cells by induction of insulin-like growth factor binding protein-5 (IGFBP-5) secretion. Biochemical and Biophysical Research Communications 239 706-709.

Hwa V, Oh Y \& Rosenfeld RG 1997 Insulin-like growth factor binding protein- 3 and -5 are regulated by transforming growth factor-beta and retinoic acid in the human prostate adenocarcinoma cell line PC-3. Endocrine 6 235-242.

Jehle PM, Ostertag A, Schulten K, Schulz W, Jehle DR, Stracke S, Fiedler R, Deuber HJ, Keller F, Boehm BO et al. 2000 Insulin-like growth factor system components in hyperparathyroidism and renal osteodystrophy. Kidney International 57 423-436.

Jenster G, Spencer TE, Burcin MM, Tsai SY, Tsai MJ \& O'Malley BW 1997 Steroid receptor induction of gene transcription: a two-step model. PNAS 94 7879-7884.

Ji C, Chen Y, Centrella M \& McCarthy TL 1999 Activation of the insulin-like growth factor-binding protein-5 promoter in osteoblasts by cooperative E box, CCAAT enhancer-binding protein, and nuclear factor-1 deoxyribonucleic acid-binding sequences. Endocrinology 140 4564-4572.

Kasper M, Regl G, Frischauf AM \& Aberger F 2006 GLI transcription factors: mediators of oncogenic Hedgehog signalling. European Journal of Cancer 42 437-445.

Kawagoe H \& Grosveld GC $2005 a$ Conditional MN1-TEL knock-in mice develop acute myeloid leukemia in conjunction with overexpression of HOXA9. Blood 106 4269-4277.
Kawagoe H \& Grosveld GC 2005 b MN1-TEL myeloid oncoprotein expressed in multipotent progenitors perturbs both myeloid and lymphoid growth and causes T-lymphoid tumors in mice. Blood 106 $4278-4286$.

Kiepe D, Ciarmatori S, Hoeflich A, Wolf E \& Tonshoff B 2005 Insulinlike growth factor (IGF)-I stimulates cell proliferation and induces IGF binding protein (IGFBP)-3 and IGFBP-5 gene expression in cultured growth plate chondrocytes via distinct signaling pathways. Endocrinology 146 3096-3104.

Kim YS, Nakanishi G, Lewandoski M \& Jetten AM 2003 GLIS3, a novel member of the GLIS subfamily of Kruppel-like zinc finger proteins with repressor and activation functions. Nucleic Acids Research 31 5513-5525.

van Kleffens M, Groffen CA, Dits NF, Lindenbergh-Kortleve DJ, Schuller AG, Bradshaw SL, Pintar JE, Zwarthoff EC, Drop SL \& van Neck JW 1999 Generation of antisera to mouse insulin-like growth factor binding proteins (IGFBP)-1 to -6: comparison of IGFBP protein and messenger ribonucleic acid localization in the mouse embryo. Endocrinology 140 5944-5952.

Knowles BB, Howe CC \& Aden DP 1980 Human hepatocellular carcinoma cell lines secrete the major plasma proteins and hepatitis B surface antigen. Science 209 497-499.

Kros J, de Greve K, van Tilborg A, Hop W, Pieterman H, Avezaat C, Lekanne Dit Deprez R \& Zwarthoff E 2001 NF2 status of meningiomas is associated with tumour localization and histology. Journal of Pathology 194 367-372.

Lekanne Deprez RH, Riegman PH, Groen NA, Warringa UL, van Biezen NA, Molijn AC, Bootsma D, de Jong PJ, Menon AG, Kley NA et al. 1995 Cloning and characterization of MN1, a gene from chromosome $22 \mathrm{q} 11$, which is disrupted by a balanced translocation in a meningioma. Oncogene 10 1521-1528.

Meester-Smoor MA, Vermeij M, van Helmond MJ, Molijn AC, van Wely KH, Hekman AC, Vermey-Keers C, Riegman PH \& Zwarthoff EC 2005 Targeted disruption of the Mnl oncogene results in severe defects in development of membranous bones of the cranial skeleton. Molecular and Cellular Biology 25 4229-4236.

Miyake H, Pollak M \& Gleave ME 2000 $a$ Castration-induced up-regulation of insulin-like growth factor binding protein-5 potentiates insulin-like growth factor-I activity and accelerates progression to androgen independence in prostate cancer models. Cancer Research 60 3058-3064.

Miyake H, Nelson C, Rennie PS \& Gleave ME $2000 b$ Overexpression of insulin-like growth factor binding protein- 5 helps accelerate progression to androgen-independence in the human prostate LNCaP tumor model through activation of phosphatidylinositol 3'kinase pathway. Endocrinology 141 2257-2265.

Mohan S \& Baylink DJ 2002 IGF-binding proteins are multifunctional and act via IGF-dependent and -independent mechanisms. Journal of Endocrinology 175 19-31.

Nordqvist AC \& Mathiesen T 2002 Expression of IGF-II, IGFBP-2, -5, and -6 in meningiomas with different brain invasiveness. Journal of Neuro-oncology 57 19-26.

Osoegawa K, Woon PY, Zhao B, Frengen E, Tateno M, Catanese JJ \& de Jong PJ 1998 An improved approach for construction of bacterial artificial chromosome libraries. Genomics 52 1-8.

Perks CM, McCaig C \& Holly JM 2000 Differential insulin-like growth factor (IGF)-independent interactions of IGF binding protein-3 and IGF binding protein- 5 on apoptosis in human breast cancer cells. Involvement of the mitochondria. Journal of Cellular Biochemistry 80 248-258.

Rosen CJ \& Donahue LR 1998 Insulin-like growth factors and bone: the osteoporosis connection revisited. Proceedings of the Society for Experimental Biology and Medicine 219 1-7.

Salih DA, Tripathi G, Holding C, Szestak TA, Gonzalez MI, Carter EJ, Cobb LJ, Eisemann JE \& Pell JM 2004 Insulin-like growth factor-binding protein 5 (IGFBP5) compromises survival, growth, muscle development, and fertility in mice. PNAS 101 $4314-4319$. 
Schneider MR, Zhou R, Hoeflich A, Krebs O, Schmidt J, Mohan S, Wolf E \& Lahm H 2001 Insulin-like growth factor-binding protein-5 inhibits growth and induces differentiation of mouse osteosarcoma cells. Biochemical and Biophysical Research Communications $\mathbf{2 8 8}$ 435-442.

Schneider MR, Wolf E, Hoeflich A \& Lahm H 2002 IGF-binding protein-5: flexible player in the IGF system and effector on its own. Journal of Endocrinology 172 423-440.

Schuller AG, Groffen C, van Neck JW, Zwarthoff EC \& Drop SL 1994 cDNA cloning and mRNA expression of the six mouse insulin-like growth factor binding proteins. Molecular and Cellular Endocrinology 104 57-66.

Shemer J, Yaron A, Werner H, Shao ZM, Sheikh MS, Fontana JA, LeRoith D \& Roberts CT Jr 1993 Regulation of insulin-like growth factor (IGF) binding protein-5 in the T47D human breast carcinoma cell line by IGF-I and retinoic acid. Journal of Clinical Endocrinology and Metabolism 77 1246-1250.

Sutton AL, Zhang X, Ellison TI \& Macdonald PN 2005 The 1,25(OH)2D3-regulated transcription factor MN1 stimulates VDRmediated transcription and inhibits osteoblastic cell proliferation. Molecular Endocrinology 19 2234-2244.

Tanno B, Negroni A, Vitali R, Pirozzoli MC, Cesi V, Mancini C, Calabretta B \& Raschella G 2002 Expression of insulin-like growth factor-binding protein 5 in neuroblastoma cells is regulated at the transcriptional level by $\mathrm{c}-\mathrm{Myb}$ and $\mathrm{B}-\mathrm{Myb}$ via direct and indirect mechanisms. Journal of Biological Chemistry 277 23172-23180.

van Wely KH, Molijn AC, Buijs A, Meester-Smoor MA, Aarnoudse AJ, Hellemons A, den Besten P, Grosveld GC \& Zwarthoff EC 2003 The MN1 oncoprotein synergizes with coactivators RAC3 and p300 in RAR-RXR-mediated transcription. Oncogene 22 699-709.

Wan YJ, Cai Y \& Magee TR 1998 Retinoic acid differentially regulates retinoic acid receptor-mediated pathways in the Hep3B cell line. Experimental Cell Research 238 241-247.

Xie X, Lu J, Kulbokas EJ, Golub TR, Mootha V, Lindblad-Toh K, Lander ES \& Kellis M 2005 Systematic discovery of regulatory motifs in human promoters and $3^{\prime}$ UTRs by comparison of several mammals. Nature 434 338-345.

Yeh LC \& Lee JC 2000 Identification of an osteogenic protein-1 (bone morphogenetic protein-7)-responsive element in the promoter of the rat insulin-like growth factor-binding protein-5 gene. Endocrinology $1413278-3286$.

Received 19 July 2006

Accepted 26 October 2006

Made available online as an Accepted Preprint on 17 November 2006 